\title{
Germanica
}

GERMANICA

31 | 2002

Le travail de réécriture dans la littérature de langue allemande au XXe siècle

\section{«La vérité de la littérature ». Intertextualités littéraires et mythiques chez Dieter Wellershoff}

"Die Wahrheit der Literatur ». Literarische und mythische Intertextualität bei

Dieter Wellershoff

\section{Gérard Laudin}

\section{(2) OpenEdition}

Journals

Édition électronique

URL : http://journals.openedition.org/germanica/2077

DOI : 10.4000/germanica.2077

ISSN : 2107-0784

Éditeur

Université de Lille

Édition imprimée

Date de publication : 31 décembre 2002

Pagination : 159-173

ISBN : 9782913857100

ISSN : 0984-2632

Référence électronique

Gérard Laudin, « "La vérité de la littérature ». Intertextualités littéraires et mythiques chez Dieter Wellershoff », Germanica [En ligne], 31 | 2002, mis en ligne le 21 mars 2013, consulté le 06 octobre 2020. URL : http://journals.openedition.org/germanica/2077 ; DOI : https://doi.org/10.4000/ germanica.2077

Ce document a été généré automatiquement le 6 octobre 2020.

(c) Tous droits réservés 


\title{
« La vérité de la littérature ». Intertextualités littéraires et mythiques chez Dieter Wellershoff
}

\author{
«Die Wahrheit der Literatur ». Literarische und mythische Intertextualität bei \\ Dieter Wellershoff
}

Gérard Laudin

1 En 2000, Dieter Wellershoff, romancier et auteur de nombreux essais théoriques sur la littérature, en particulier dans ses rapports avec le temps présent ${ }^{1}$, accède à une large consécration avec Der Liebeswunsch, un roman dont le titre reflète une réflexion qui occupe ses écrits depuis de longues années: la quête du bonheur, expression de l'«appétit de vivre " ${ }^{2}$, est devenue un jeu d'autant plus dangereux que le désir d'épanouissement individuel est désormais une nouvelle contrainte sociale à laquelle le psychisme souvent profondément altéré de l'homme moderne ne peut guère faire face : «Glücklichsein, zum allgemeinen gesellschaftlichen Gebot erhoben, wird damit auch zum hochriskanten Spiel »3. Wellershoff écrit lui-même à propos de la thématique de Der Liebeswunsch :

Das sind die beiden Eckpunkte der fundamentalen Exzentrizität der menschlichen

Existenz: Getrieben von Leidenschaften, Wünschen, Ängsten und alltäglichen Notwendigkeiten handeln und irren wir in der halbblinden Unmittelbarkeit, die Ernst Bloch das « Dunkel des gelebten Augenblicks » genannt hat ${ }^{4}$.

2 Dans ce roman, Wellershoff décrit avec sa coutumière précision les tensions qui naissent peu à peu entre des êtres, les petits détails en apparence insignifiants qui anéantissent progressivement une relation, les "petites perceptions $»^{5}$ quasi leibniziennes et les choses indécises (récurrence de l'adjectif "unbestimmt»), les signes à peine perceptibles par lesquels les doutes mais aussi les certitudes s'insinuent $(L W, 168)$. À côté de passages d'une grande précision documentaire (une opération chirurgicale, un procès), domine une approche phénoménologique qui préfère décrire les gestes dont se déduisent les sentiments que les sentiments eux-mêmes. Des passages fréquents de la première à la troisième personne, les interventions des différents 
personnages à tour de rôle dans le récit, ce que Wellershoff appelle « ein Zoomen von der allgemeinen zur individuellen Perspektive : was man vom Film her kennt $»^{6}$, créent un "perspectivisme dialogique", isomorphe à la vérité éclatée dont il veut rendre compte, une expression utilisée par un des personnages à propos de son métier de juge : « das Dialogische und Perspektivische der Urteilsbildung » $(L W, 209)$.

Dans Der Liebeswunsch, Anja, une étudiante de 29 ans, fait la connaissance de Leonhard, ami d'un couple de médecins, Paul et Marlene, dont elle garde la villa durant leurs vacances. Anja et Leonhard se marient sans tarder. Entre ces quatre personnages, qui se rencontrent régulièrement, se développe une sociabilité qui toutefois cache mal les ambiguïtés sur lesquelles se fondent leurs relations et les disharmonies naissantes dans le jeune couple. Déçue par la froideur toute bureaucratique de son mari, qu'elle a de toutes façons épousé faute de meilleure perspective, Anja se laisse séduire par Paul, en qui elle voit un homme aimable et attentionné, sans savoir qu'il avait déjà dans le passé séduit puis épousé Marlene, alors qu'elle était l'amie de Leonhard. Trompé par Anja, celui-ci demande immédiatement le divorce, Marlene se sépare de Paul qui plus tard rompt avec Anja. Incapable de faire face à une situation trop complexe pour elle, Anja sombre d'abord dans l'alcoolisme avant de se suicider en se laissant tomber du haut d'un immeuble, comme sa presque homonyme, l'Anna Karénine de Tolstoï (1878), elle aussi adultère, se jette d'un train.

Dans ces constellations évolutives de personnages («Wir waren vier Figuren auf einem engen Spielfeld», $L W, 155)$, on aura reconnu des linéaments rappelant les Wahlverwandtschaften de Goethe. De fait, outre la thématique générale de la recomposition de couples dans le cadre d'un "Bildungsroman", de menus détails rappellent Goethe, comme le fait que le processus de dislocation du jeune couple se trouve engagé après un accident survenu à leur enfant et dont Leonhard juge Anja responsable. Comme Die Wahlverwandtschaften, Der Liebeswunsch repose sur un jeu de symétries et de contrastes entre les personnages. En face de Marlene, indépendante et sûre d'elle-même, qui fait une brillante carrière, Anja, sans situation professionnelle, est désemparée et faible, totalement dépendante de Paul et animée d'un désir naïf de bonheur qui causera sa perte. Face à Paul, le séducteur sans scrupules, qui trompe à deux reprises son meilleur ami et qui se montre finalement faible dès que le drame se noue, Leonhard, mû d'un grand besoin de certitudes ( $L W, 192)$, maître de soi et psychorigide, politiquement conservateur, a bien intériorisé les principes éthiques des stoïciens qu'il lit, mais sa vie privée est un échec aussi patent que sa vie professionnelle de juge est réussie.

Outre ces détails, la relation avec Die Wahlverwandtschaften est suggérée par le fait que Wellershoff, qui a consacré de nombreuses études aux romans du xIX siècle, écrit dans l'essai déjà cité, Der Treibsand der Gefühle, dont Der Liebeswunsch reprend les perspectives :

Das schrecklichste Beispiel verinnerlichter Schuld gibt Ottilie in den Wahlverwandtschaften, die sich selbst zum Tod durch Verhungern verurteilt, nachdem sie das Kind der Ehefrau ihres Geliebten in einer aus tiefstem Unbewussten stammenden Verwirrung hat ertrinken lassen $(T G, 635)$.

6 Toutefois, cette référence sert à mieux faire ressortir ce qui distingue les relations amoureuses au XIX ${ }^{e}$ siècle et aujourd'hui :

Damals liefen die Liebenden gegen die Wände der Institutionen, heute waten sie im Sumpf einer verschwommenen Glücksideologie (TG, 641).

Das individuelle Glücksstreben scheitert nicht mehr an der Härte des 
gesellschaftlichen Widerstandes und der Enge der herrschenden Moral, sondern an seiner eigenen Ideologisierung ( $T G, 635)$.

7 En lieu et place de la faute et du remords qui structurent le psychisme des personnages de Goethe, cette idéologie du bonheur reflète celle de la société moderne asservie aux valeurs de la concurrence et du succcès $(T G, 639)$. Le principe de performance (« das Leistungsprinzip ", TG, 636) s'exprime ici aussi sous le « masque d'une invitation à jouir pleinement de la vie » ("maskiert als Aufforderung zum vollen Lebensgenuss », TG, 636) dans une société où le marché et ses lois s'étendent aux relations entre les gens :

Ziel scheint die chancengleiche Internationale der freien Sexualpartner zu sein, die (...) sich zu einem riesigen sexuellen Tauschmarkt entwickelt hat $(T G, 636)$.

Il n'est pas étonnant dès lors que l'hédonisme n'ait pas que des couleurs chatoyantes. Le narcissisme qui le sous-tend génère des troubles psychologiques gravissimes:

Diese vermeintliche Demokratie des Konsums und der unbeschränkten Selbstverwirklichung erzeugt natürlich massenhaft Verlierer und treibt sie als Gestörte in den Alkoholismus, die Depression und die Therapiegruppen (TG, 637).

Il en résulte que le mode d'intériorisation de la norme sociale et partant de l'échec ont changé radicalement entre l'époque de Die Wahlverwandtschaften et la nôtre :

Der äussere Zwang, überwunden durch Aufklärung und Emanzipation, wandert nach innen und entsteht dort neu (TG, 641),

10 Cet impératif social place l'individu seul devant ses échecs et transforme en vaincu celui qui ne parvient pas au bonheur auquel il aspire. Wellershoff montre certes ainsi, comme le suggère Claudine Borries ${ }^{7}$, ce que le public allemand vient de découvrir avec enthousiasme chez Michel Houellebecq, mais il se place dans une perspective de critique de l'ordre social et du jeu de la concurrence dans le monde marchand, loin des poncifs décadentiels et des accusations aussi complaisantes que plates contre les pères de la « postmodernité » sur lesquels se fonde le romancier français ${ }^{8}$.

11 L'(al)chimie des affinités, que Goethe emprunte au chimiste suédois Bergmann, est remplacée par une perspective psycho-sociologique. Si le drame futur est contenu en germe, comme chez Goethe, dans des déséquilibres constitutifs, il n'est pas indifférent qu'une de ses causes principales tienne au fait qu'Anja, psychologiquement moins mûre et ainsi plus fragile que les autres (tout comme Ottilie chez Goethe), demeure socialement et culturellement étrangère dans ce groupe des trois amis, ce qui provoque son isolement. Le décalage culturel apparaît dès le voyage de noces en Italie de Leonhard et Anja, marqué par un mélange de froideur distante et de désaccord profond par dissemblance de goûts $(L W, 52,54,64)$. La crise révèle ainsi une harmonie de pure apparence et la fragilité de l'union d'Anja et de Leonhard dans laquelle il entre peu d'inclination véritable. En se mariant, Anja avait recherché une position sociale enviable, et Leonhard avait plus ou moins consciemment cherché à compenser l'échec subi avec Marlene et à assainir les relations avec ses amis :

Er, der in unserem Freundschaftsbund einmal der Verlierer gewesen war (...) hatte Symmetrie hergestellt und damit ganz neue Möglichkeiten des freundschaftlichen Umgangs geschaffen $(L W, 75)$.

12 Leonhard est incapable de comprendre la fragilité d'Anja. Au début, il l'intimide, puis l'aversion succède à l'admiration face à un mari qu'elle croit totalement dépourvu de sensibilité. Frustrée dans ses relations avec Leonhard, Anja s'éprend de Paul avec d'autant plus de violence. Comme les êtres dont les troubles sont décrits dans Der Treibsand der Gefühle («wer bewusst oder unbewusst einen existentiellen Mangel 
empfindet, wer also Trost, Kompensation, Bestätigung und Bestärkung sucht (...) », TG, 639), elle croit pouvoir s'abandonner à ses sentiments troubles et assouvir avec lui son désir d'amour, cet « aveugle appétit de vivre » («blinder Appetit des Lebens » (GS, 404) déjà évoqué, qui donnerait à sa vie une certaine densité. En effet, toute sa vie, y compris érotique, paraît se dérouler à une certaine distance d'elle-même, elle donne un impression d'apesanteur qu'elle déplore elle-même ( $L W, 73)$, «manque de contours » (« die Undeutlichkeit ihrer Person », $L W, 73$ ).

13 Cette "sensualité de somnambule» («schlafwandlerische Sinnlichkeit», LW, 156) conduira au tragique en entraînant quatre personnes dans le malheur, l'autodestruction pour Paul $(L W, 362)$ et la mort pour Anja. En effet, façe à ces personnages chez qui le désir d'amour est contrebalancé par la maîtrise de soi, Anja représente la variante ouvertement névrotique et peu contrôlée de ce désir, de sorte que « son fol espoir " ${ }^{9}$ fait éclater le cadre de cette relation à quatre, asymétrique et peu viable, par la violence de son élan sexuel pour Paul qu'elle attend toujours au lit presque en état de trance.

14 Fait aggravant, son « insatisable désir d'amour $»^{10}$, elle le formule de la manière la plus platement romantique $(L W, 269)$ dans une sorte de sentimentalisme de "Trivialliteratur ». L'attente et la rhétorique d'Anja sont étrangères aux catégories de Paul qui n'y voit qu'une pathologie à laquelle il est prudent de se soustraire :

Der Liebeswahn dieser Frau war eine Falle. (...) Das waren doch alles nur Worte, an denen sie sich berauschte, weil sie süchtig nach großen Gefühlen war $(L W, 277)$.

Sa sensibilité et son idéologie proviennent du roman à l'eau de rose dont se moque Wellershoff :

Nur noch im Kitschroman laufen die Liebenden nach der unruhigen oder stürmischen Zeit der Partnersuche in den sicheren Hafen der Ehe ein (TG, 637).

Le choix de la référence intertextuelle à un roman célèbre de Goethe, même dans une perspective marquant plutôt la distance que la proximité, n'a pas une simple finalité de jeu intellectuel. Wellershoff, sceptique tout autant envers la littérature engagée comme la comprend Enzensberger qu'à l'égard de la «littérature métaphysique » ( " metaphysische Literatur, die immer noch universelle Daseinsmodelle und Sinnbilder, wenn auch vielleicht der Sinnferne schafft $»^{11}$ ), voit dans la littérature un possible instrument d'appréhension du monde, aux limites du sensible et du discursif («Immer wieder versuchen die Schriftsteller neue empfindliche Grenzwerte der Erfahrung zu erreichen » $(G S, 407)$, une perspective générale que reflète les titres de plusieurs essais : Die Wahrheit der Literatur ${ }^{12}$ ou Der Roman und die Erfahrbarkeit der Welt (1988).

17 Cette perspective, la recherche d'un lien unissant la littérature, les arts et la vie pratique $^{13}$, Wellershoff la réaffirme, à propos de Der Liebeswunsch :

[Wir] sind fähig, zu uns selbst auf Distanz zu gehen und uns aus einer Ferne zu betrachten, in der die Gründe unserer Aufregungen unerheblich und unsere Getriebenheiten und Anstrengungen absurd erscheinen. Diese Spannung des doppelten, gespaltenen Blicks von nah und fern, das Dazwischen-und-darüber-sein ist unsere größte existenzielle Erkenntnischance. Sie wird in der Literatur auf vielfache Weise erlebbar, etwa wenn konkurrierende personale Perspektiven sich wechselseitig in ihrer Partikularität zu erkennen geben oder wenn das Kontinuum der Handlung durch ein unerwartetes Ereignis unterbrochen wird. Plötzlich eröffnet sich die Erkenntnischance eines Blicks aus der Distan $z^{14}$.

18 Toutefois, ces relations intertextuelles aux Wahlverwandtschaften, authentifiées par un texte théorique avec lequel Der Liebeswunsch, comme il est fréquent chez Wellershoff, 
s'inscrit dans un jeu d'échos, ne constituent guère qu'un cadre assez vague, tout comme demeure superficielle l'allusion au mythe de la Méduse dans le récit Zikadengeschrei (1995), qui analyse la fascination exercée sur un homme par une femme dont le visage est très déformé. C'est en revanche une intertextualité beaucoup plus précise qu'on trouve dans Die Sirene (1980). Cette nouvelle ne reprend pas seulement, comme l'ont fait déjà Jean Cocteau dans La voix humaine (1930) et Michel Tournier dans Tristan Vox ${ }^{15}$, le motif de la fascination exercée par la voix d'une inconnue, perçue par une technique moderne de communication à distance, ici le téléphone, mais inaccessible au regard. Revisité par les perspectives de l'anthropologie sociale, ce motif a occupé Dieter Wellershoff à de nombreuses reprises. Il y fait de nombreuses allusions dans son œuvre théorique, condensées dans l'essai Der Gesang der Sirenen (1973) ${ }^{16}$, lequel s'appuie sur le chapitre «Odysseus oder Mythos und Aufklärung » de Dialektik der Aufklärung d'Adorno et Horkheimer ${ }^{17}$, ainsi que l'essai de Maurice Blanchot, «Le Chant des Sirènes », dont un passage est cité en exergue de Die Sirene ${ }^{18}$.

De Blanchot, Wellershoff reprend l'idée que le chant ne manifeste pas seulement une puissance extérieure exerçant sur l'homme une attraction irrésistible (motif de la séductrice fatale), mais qu'il est bel et bien une force intérieure à l'homme, lequel toutefois ne l'identifie pas immédiatement comme telle. L'audition du chant des sirènes rencontre ainsi l'imaginaire (" die Begegnung mit dem Imaginären », GS, 398), la zone de la conscience enfouie.

Der Mythos vom Gesang der Sirenen, der die Seefahrer in den Tod lockt, beschreibt diesen Vorgang. Die Seefahrer, die sich von der Sicherheit der Küsten, vom festen, verbürgten Boden gelöst haben und das weite, anhaltslose Meer befahren, beginnen die Stimmen des eigenen Inneren zu hören wie beschwörende Einflüsterungen, die die Lüste der Regression, der Entgrenzung und des Ichverlustes versprechen ${ }^{19}$.

Dans la légende, y compris dans la variante qu'en donnent Brentano et Heine avec la Loreley, la beauté du chant des sirènes, génératrice de plaisir, entraîne la mort. Chez Homère, Ulysse triomphe des sirènes grâce à une ruse qui le sauve. Wellershoff reprend, ainsi que le montre Alo Allkemper ${ }^{20}$, les fondements de l'interprétation qu'Adorno et Horkheimer proposent du récit homérique. La ruse d'Ulysse y est analysée comme prototypique de la rationalité calculatoire («kalkulatorische Rationalität») en ce qu'elle symbolise un paradoxe fondamental de la civilisation occidentale: l'homme ne sauve sa vie qu'en sacrifiant sa vie dans le cadre d'une dialectique de la conservation de soi et du renoncement: «die Paradoxie des Opfers, Leben zu erhalten durch das Opfer von Leben, die blutige Rationalität, Prospekt aller Humanität, zur Rationalität der Entsagung $»^{21}$. Pour Wellershoff, la société, « ce système de sauvegarde collective » («Gesellschaft, das System kollektiver Lebenserhaltung »), fonctionne en raison même de cette rationalité calculatoire, de même que la vie pour se maintenir opprime la vie, "im Namen der Lebenserhaltung Leben unterdrückt und beschädigt und vielleicht um seinen Sinn betrügt ${ }^{22}$. En se faisant lier au mât, Ulysse refuse de se soumettre pleinement à la puissance des sirènes, il définit lui-même les limites de son abandon au plaisir et maîtrise ainsi sa nature et ses instincts. L'intériorisation de son renoncement va de pair avec l'assouvissement du désir : il écoute les sirènes, mais dans les chaînes.

21 Dans Gesang der Sirenen, Wellershoff reconstitue cette dialectique en soulignant l'importance du langage. L'« aveugle appétit de vivre », directement lié à l'imaginaire, se trouve soumis à l'ordre social qui l'opprime et le contraint à la sublimation, laquelle 
passe par le langage de l'abstraction et de la rationalité. Le monde, une fois rationalisé, perd son caractère dangereux métaphorisé par le chant des sirènes :

Der Gesang der Sirenen verstummt im Herrschaftsbereich der rationalen Sprache, der Fachsprache, der definierten Allgemeinheiten $(G S, 135)$.

Le langage de la rationalité permet à l'homme de se créer le rapport au monde dont il a besoin, fondé sur un ordre qui le rassure. Mais quand il y parvient par la maîtrise de soi et du monde, cet ordre menace alors de l'étouffer et le plonge dans la solitude, un motif récurrent chez Wellershoff dont bien des personnages sont en crise ${ }^{23}$.

L'ambivalence de cette expérience, de «la rencontre avec l'imaginaire », le plaisir et ses dangers, est le thème du roman Die Sirene dont le héros, un professeur de pédagogie qui a pris un semestre sabbatique pour être libéré du quotidien et écrire un livre, reçoit un appel téléphonique d'une inconnue aux abois. Cette voix, qui demeurera sans visage, fait surgir en Elsheimer des pulsions enfouies, une libido qui paraissait sous contrôle, tant il est vrai que l'imaginaire, si l'on en croit Bachelard, est moins «la faculté de former des images » que « la faculté de déformer les images fournies par la perception » ${ }^{24}$.

Cette « sirène ", qui renouvelle ses appels plusieurs fois par jour, est fort ambivalente : psychologiquement très affaiblie, elle prend conscience de la puissance qu'elle exerce sur Elsheimer, ce qui la rattache au type de la femme fatale. Leurs conversations déclenchent en lui un processus qui va révéler l'étroitesse et finalement le vide de son existence où chaque chose et chaque être a sa place et, comme dans Der Liebeswunsch, la fragilité de l'harmonie de cet univers en apparence lisse et ordonné dans laquelle il vit avec sa famille. Il apparaît ainsi brutalement qu'il est étouffé par ce monde tranquille, mais également triste et solitaire, qu'il s'est lui-même construit.

Très vite, Elsheimer commence à ressentir des signes d'insatisfaction existentielle, à reconnaître que quelque chose dans sa vie sonne faux. Le premier symptôme est l'angoisse de la page blanche. Il est, sans raison apparente, incapable de formuler, de penser, de rédiger. Il sombre dans un activisme mal contrôlé ou bien erre dans la ville sans but précis, en se sentant étranger. Sous l'influence de la "sirène ", qui comme le personnage féminin de $L a$ Voix humaine de Cocteau, n'a aucune activité («Sie hatte sogar ihre Arbeit aufgegeben und lebte nur noch neben dem Telefon", DS, 14), le professeur Elsheimer, dont toute l'existence était ordonnée autour de sa vie professionnelle et familiale, se détache partiellement de l'ordre social. L'image de l'homme enchaîné à son rôle professionnel et social devient ici un embrayeur plaisant d'intertextualité :

Etwas ist falsch gewesen, seit langem. Er hatte auf Verlust, auf Verminderung gelebt, ohne es zu wissen. Er hatte gelebt wie alle anderen, wie seine Kollegen, die an ihre Berufe und an ihre Ehen, an ihre Erfolge, Probleme und Begriffe gekettet waren wie an Ruderbänke (DS, 89).

Comme chez Adorno, la crise, à la fois salutaire et dangereuse, fera échapper Elsheimer à la pression qui l'entoure, mais en même temps représentera une menace pour lui. Un important signe de cette crise, telle que Wellershoff la décrit dans Der Gesang der Sirenen, est le fait que le monde paraisse soudain pesant :

Eine Verwandlung kündigt sich an, spürbar erst daran, dass sich der Druck der Welt auf das Ich verstärkt und dessen Einsamkeit sich vertieft (GS, 399).

La seconde étape, de prime abord positive celle-ci, est la promesse d'une vie plus intense dès lors que le sujet consent à s'abandonner à la stimulation érotique que 
procure la voix ${ }^{25}$. Cette intensité nouvelle est d'abord sensorielle. Elsheimer redécouvre son corps, il se perçoit lui-même et perçoit le monde avec beaucoup plus d'acuité : il observe, est attentif aux sensations (DS, 46), il note que ses énergies se décuplent (DS, 92, 93, 115), qu'une tension permanente parcourt son corps (DS, 82, 100, 108). Cette découverte de soi est liée à la découverte de l'autre, puisque l'autre, la voix de l'autre, est en fait l'autre soi-même. Comme dans l'interprétation de Blanchot, cet appel de l'imaginaire est la métaphore de la voix de l'autre en soi, du moi longtemps occulté. Elsheimer remarque que cette voix qui promet la vie sous la forme de l'expérience sensorielle intense est en fait partie de son moi (DS, 70).

L'instant durant lequel l'homme, fatigué de sa vie quotidienne, perçoit les choses autrement, peut être décrit, sur le mode métaphorique, comme le signe de sa capacité à entendre de nouveau le chant des sirènes :

(...) die chimärischen Stimmen der Sirenen sprechen eine dunkle, dem Ich nicht völlig verständliche Sprache $(G S, 399)$.

Comme Blanchot, Wellershoff considère que cette capacité recouvrée à percevoir est une nouvelle chance dans la vie : ce n'est que par le biais d'une crise existentielle que l'homme est capable de scruter son existence avec davantage de sagacité. Il en résulte un moment d'une grande richesse,

einen Augenblick, in dem die gewohnten Verschränkungen von Subjekt zum objekt, Innen- und Aussenwelt ins Wanken geraten, und die Möglichkeit einer neuen Zuordnung sich zeigt. Das kann erlebt werden als eine leise Irritation, ein Befremden, ein Aufleuchten neuer, individueller Lebensmomente und bisher nicht wahrgenommener Züge der Realität, aber eben auch als eine tiefe, umwälzende Krise. Ein katastrophaler Orientierungsverlust kann drohen. Aber es können sich auch neue Möglichkeiten des Lebens öffnen. Der Beginn von etwas Neuem ist immer ein Dissoziationsprozess (EE, 541-542).

Dans ces moments, les choses les plus banales retrouvent un sens :

Das Alltägliche wird wieder auffällig, das Selbstverständliche wird sonderbar, im täglich Gesehenen zeigt sich etwas Ungesehenes, das Bekannte stellt sich als das Unbekannte heraus, und so entsteht ein Unsicherheitszustand, dessen erregende Lust-Angst-Tönung ein Vorgefühl von Freiheit ist. Die Welt erscheint plötzlich wieder als ein offenes Feld lockender und auch gefährlicher Möglichkeiten, sie wird angeleuchtet von bisher unterdrückten oder kulturell heruntergestimmten Wünschen, die nun wieder frei zu vagieren beginnen ${ }^{26}$.

La thématique de Die Sirene est anticipée dans un essai déjà cité de 1978 :

Ich möchte ein Beispiel dafür anführen, die Beschreibung eines typischen, krisenhaften Initialmoments. Nehmen wir an, ein Mann (...) unterbricht eine lange anstrengende Autofahrt, um in einem Gasthof eine Tasse Kaffee zu trinken (...). Plötzlich tut sich ein tiefer Widerspruch auf zwischen ihm und seiner Umgebung, aber auch zwischen ihm und der Welt und ihm jetzt, dort am Tisch, und seinem bisherigen Leben. Auf einmal sieht er alles anders, in einem neuen, befremdenden Licht: er kann nicht mehr so weitermachen. (...) Das könnte der Anfang eines Romans sein, der von einer Identitätskrise handelt (EE, 542).

Pour qu'un tel processus de prise de conscience ait lieu, l'individu doit être poussé à ses limites extrêmes. C'est précisément ce qui s'est produit avec le premier appel téléphonique de la "sirène ». Dès qu'il décroche l'écouteur, c'est un mécanisme d'aliénation quasi hypnotique qui se déclenche et qui conduira à une fissure dans sa personnalité : «Es gab nun zwei Welten, in denen er lebte» (DS, 94). Il ressent une " paralysie » ("Lähmung »), qui le conduit à obéir à cette voix. Il devient un automate, «behext und ohnmächtig» (DS, 92), jusqu'à ce que la femme raccroche : «Erst als sie 
auflegte, löste sich die Lähmung und er fand zu sich zurück» (DS, 28). Elsheimer effectue même une sorte de régression symbolique vers un équivalent fotal: "Den Abend über hatte er an der Phantasie festgehalten, sich im Innern eines mächtigen Schiffes zu befinden, das ruhig durch das nächtliche Meer fuhr » (DS, 79).

Selon une thèse centrale de Wahrnehmung und Phantasie, perception et imagination sont deux principes opposés mais qui se complètent. La voix finit par avoir une présence quasi physique (DS, 100). Pour Elsheimer, la perturbation est telle que le réel et l'imaginaire, l'extérieur et l'intérieur se confondent :

Es gab ihm die Empfindung, als drücke ihn jemand mit hohlen Händen sanft auf beide Ohren und mache es ihm schwer zu unterscheiden, was innen und aussen war (DS, 79).

Doch er kam nicht zu sich selbst, sondern fühlte, dass er in eine wesenlose Unbestimmtheit abglitt, in eine betäubte innere Stille, die ihn konfus machte, als dächte er gleichzeitig in verschiedene Richtungen, verlöre sich aber überall in diese Spurlosigkeit (DS, 25).

34 Toutefois, alors que dans Der Liebeswunsch, l'intensification du vécu précède l'autodestruction des personnages, Elsheimer sortira altéré de cette expérience, mais reprendra pied. Partiellement provoquée par des épreuves sans rapport avec la «sirène » (difficultés avec ses enfants, mort d'un collègue...), l'intensification de la crise, liée à l'irrépressible désir de parler à cette femme, puis de la rencontrer, provoque chez cet homme habituellement très maître de soi un sursaut :

Ich bin es nicht, dachte er (...) dieser Verrückte, der bereit war, alles aufs Spiel zu setzen und zu zerstören, um jetzt sofort mit dieser Frau zu sprechen, und dass es gerade die Zerstörung war, die er suchte, der er sich ergeben wollte, als enthielte sie ein ungeahntes Versprechen (DS, 111).

Les appels de la sirène ayant réveillé sa libido, il se met à s'intéresser aux prostituées, qui exercent sur lui la même fascination que la voix. Il réalise avec l'une d'elle, qui est presque une enfant, la double transgression du tabou sexuel et du tabou social. On peut dire avec Alo Allkemper que la visite d'Elsheimer à la maison close est «die Degradation der erotischen Phantasie zur Hurenwirklichkeit. In seinem Bordellbesuch 'tötet' Elsheimer daher die erotische Macht der Stimme und 'verhöhnt' sie zudem, um sich endgültig zu befreien ${ }^{27}$. Cette jeune prostituée joue le rôle de l'intermédiaire entre l'imaginaire et la réalité, mais la démythification de l'imaginaire qui en résulte donne à Elsheimer le courage de résister. Quand la sirène appelle de nouveau, il couvre sa voix avec de la musique, comme Ulysse a bouché les oreilles de ses compagnons avec de la cire $(D S, 161)$.

Toutefois, l'absence de la voix ne le délivre pas. Il ne reste que « le silence des sirènes ", que Kafka juge encore plus effrayant que leur chant ${ }^{28}$ : «Warum fühlte er sich so leer?»(DS, 162). Elsheimer ne parvient à sauver sa vie qu'en détruisant les promesses d'un vécu d'une intensité quasi illimitée.

Vu la relation de réciprocité entre l'expérience traversée par Elsheimer et le sujet du livre auquel il travaille, il n'est pas étonnant que parallèlement à sa crise d'identité, il éprouve les plus grandes peines à thématiser ses idées. Il change de titre et donc de sujet (DS, 29), et s'intéresse désormais aux relations entre le moi et les autres, une perspective absente de son projet initial. Le livre qu'il projette désormais d'écrire portera sur la formation de l'identité individuelle, «Selbsterkenntnis oder über die Entstehung des Ichs » $(D S, 28)$. 
Ce qui est propre à Wellershoff dans la réinterprétation du mythe de la Sirène, c'est la mise en relation avec l'écriture, explicite à la fin de l'essai Der Gesang der Sirenen :

Diese Bilder der Todesnähe und der Einsamkeit, in denen die Schriftsteller ihre gesellschaftliche Entfremdung darstellen, üben eine tiefe Faszination auf sie aus. Sich so isoliert zu sehen, wird geradezu die Ausgangslage der Inspiration (GS, 409).

Quand la vie, dans la société, commence à perdre de sa vigueur, la littérature, la poésie, l'art ouvrent alors, grâce à la dialectique du prosaïque et du poétique, «Widerspiel von Versachlichung und Poetisierung $»^{29}$, d'autres champs que le pur " principe de réalité ». La littérature est alors l'expression d'un élan vital utopique issu des énergies pulsionnelles enfouies (« libidinöse Energien», GS, 405), dont l'expression érotique est ici la métaphore. L'art devient ainsi le lieu où se projettent les visions alternatives de la réalité ${ }^{30}$, et la poésie l'instrument d'une expérience élargie de soi, «erweiterte IchErfahrung $»^{31}$ :

Alle ihre [der Literatur] strukturellen Operationen kann man beschreiben als Angriffe auf das im Bewusstsein festsitzende Realitätsprinzip (GS, 407).

Peut-on dire qu'Elsheimer échoue, parce qu'il n'est pas un artiste, mais un commentateur ${ }^{32}$, la création scientifique représentant un substitut mineur de création littéraire ? On notera qu'en cela, Elsheimer rejoint Anja, l'étudiante de Der Liebeswunsch, qui éprouve aussi en rédigeant son mémoire de maîtrise la stérilité du chercheur ( $L W$, 38). Et il est hautement significatif, et quelque peu cocasse, qu'Elsheimer, professeur de pédagogie et spécialiste reconnu et médiatisé des modèles de communication, souffre de difficultés à communiquer. Pour cet homme qui prend son métier au sérieux, qui élabore des théories et classifie, la langue est un facteur d'ordre, apte ainsi à la formulation des idées scientifiques, mais plus largement aussi, elle est une instance de régulation, un dispositif conservateur de maintien des règles sociales. L'inexprimable n'a pas de place dans ce système.

En fait, la langue d'Elsheimer, loin d'être encore un instrument efficace de conceptualisation, s'est rigidifiée. Elle n'est plus constituée que de concepts mis bout à bout. Quand il écrit ses textes, il cherche le mot exact, et s'il s'étonne que les solutions trouvées lui paraissent toujours inappropriées, c'est qu'il ne reconnait pas que le langage n'est pas isomorphe à l'expérience, ainsi que le souligne Wellershoff dans Der Gesang der Sirenen : "wie ohnmächtig die Sprache gegenüber dem Erlebnis ist 》 (GS, 402).

2 Si la seule protection comme l'imaginaire est le savoir (GS, 399), à l'inverse savoir nommer les choses finit paradoxalement par appauvrir l'expressivité :

Die Ausdruckskraft verschwindet mit den Bildern. Sie wird ersetzt durch Benennbarkeit (...) Die Begriffe, die an der Stelle der Phantasmen treten, gehören einer sprachlichen Verkehrsform an, die das Erlebnis schon erledigt hat, und es als benennbar und erklärbar unter Kontrolle hält $(G S, 401)$.

L'incapacité à exprimer et le déficit d'expressivité engendrent le développement d'images échappant au contrôle du conscient. Wellershoff, qui s'inspire des théories de la communication formulées entre autres par Blanchot et les nouveaux romanciers, s'appuie aussi sur la thèse d'Alfred Lorenzer citée dans Der Gesang der Sirenen :

Traumbilder und neurotische Symptome [sind] Elemente einer zerstörten Sprache. In ihr äussern sich die abgesprengten Persönlichkeitsteile mehrdeutig und lückenhaft $(G S, 401)$. 

réinterprétation du mythe d'Ulysse et des sirènes un cadre conceptuel, c'est dans le cas de la référence à Die Wahlverwandtschaften un écart qui se mesure, celui séparant le roman moderne du roman européen du xix siècle, objet de nombreuses études de Wellershoff en partie réunies dans Der Roman und die Erfahrbarkeit der Welt. Toutefois, pas plus dans Die Sirene que dans Der Liebeswunsch, on n'a affaire à la simple réécriture d'un « mythe » littéraire, mais bien plutôt à une pensée qui ne s'élabore ni ne s'enrichit jamais sans références permanentes à la «Weltliteratur » et aux grands textes de la philosophie avec laquelle elle est en dialogue permanent. De ce dialogue résultent tant des essais de réflexion théorique que des productions littéraires qui constituent avec les "thèmes ", " mythes » ou "modèles " sur lesquels ils s'appuient un vaste espace d'intertextualité. Ces textes de référence servent de point d'appui à une pensée éminemment dialectique, qui fonde la réflexion de Wellershoff sur le mythe de la sirène, où la perte de soi est la condition de régénération de soi, mais qui fournit aussi le thème de Der Liebeswunsch qui entend montrer comment la recherche du bonheur engendre une contrainte dans laquelle le bonheur se perd.

\section{NOTES}

1. Né en 1925, ses premières œuvres littéraires datent des années 1960. Il est l'auteur d'une thèse : Gottfried Benn - Phänotyp dieser Stunde (Köln, 1958).

2. «Appetit des Lebens", in: Der Gesang der Sirenen (1973), désormais: GS; reproduit in: Wahrnehmung und Phantasie. Essays zur Literatur (1987); repris in : Werke, t. 4, p. 398-410; ici p. 404.

3. Der Treibsand der Gefühle und die Freiheit, glücklich zu sein (1986), (désormais : TG), in : Werke, 1997, t. 4, p. 634-643; ici p. 643.

4. Dieter Wellershoff, Rede zur Verleihung des Joseph-Breitbach-Preises, in : http : //www. adwmainz. de/joseph-breitbach/stiftung/wellershoff. htm.

5. Der Liebeswunsch, Köln (Kiepenheuer \& Witsch), 2000, désormais cité $L W$, d'après la $7^{\mathrm{e}}$ édition (2001), ici p. 200-201.

6. http://www.swr.de/bestenliste/rezension/archiv/2000/12/r4.html.

7. http://www.buchkritik.at/kritik.asp.IDX=934.

8. Michel Houellebecq, Les particules élémentaires (1998), réédition «J'ai lu», 2001, cf. en part. p. 314.

9. « Anjas Wunsch und Wahn », Der Spiegel, 30.10.2000, n44.

10. Claudine Borries, in : http://www.buchkritik.at/kritik.asp.IDX=934.

11. Wiederherstellung der Fremdheit, in : Wellershoff, Literatur und Veränderung (1962), repris in : Werke, éd. citée, t. 4, p. 233.

12. Die Wahrheit der Literatur. Sieben Gespräche, München: Wilhelm Fink Verlag 1980, en particulier les chapitres «Literatur als Erfahrung ( (p. 69-83) et « Die Wahrheitsbedingungen der Literatur» (p. 139-179).

13. Hans Helmreich, Dieter Wellershoff. Verlag Beck, Verlag edition text + kritik, München 1982, donne un aperçu précis de cette problématique dans le chapitre «Die Essays: Vom Zusammenhang zwischen Literatur, Kunst und Lebenspraxis », p. 25-34. 
14. Dieter Wellershoff, Rede zur Verleihung des Joseph-Breitbach-Preises (note 4).

15. In : Le Coq de bruyère (1978). Sur ces deux textes, cf. Jürgen Ritte, «Die Erotik des Radios. Versuch über Tourniers Novelle Tristan Vox », in : Peter-E. Knabe (Hg.), Michel Tournier, Cologne (dme) 1987, p. 167-174.

16. Cf. note 2 .

17. 1969, cité d'après l'éd. Fischer Verlag (1994), p. 50-87.

18. In : Le livre à venir, Paris 1959 ; trad. allemande 1962.

19. Erkenntnisglück und Egotrip (1978-79), désormais EE, repris in : Das Verschwinden im Bild. Über Blendwerke und Fiktionen (1980) ; in : Werke, éd. citée, t. 4, p. 543.

20. Alo Allkemper, "'An den Rändern des Bewusstseins'. Zu Dieter Wellershoffs Sirene », in : Manred Durzak/Hartmut Steinecke/Keith Bullivant, Wellershoff. Studien zu seinem Werk, Köln 1990, p. 129-144.

21. Ibid., p. 130.

22. Zukunft und Tod (1974), repris in: Werke, t. 4, p. 414.

23. Cf. Walter Oma, «Vereinsamung und Selbstzerstîrung. Dieter Wellershoff als Phänomenologe gegenwärtiger Einsamkeitsproblematik», in: Wellershoff, Studien zu seinem Werk (note 20), p. 89-111.

24. Gaston Bachelard, L'Air et les songes, Paris 1943, cité d'après rééd. Paris (José Corti) 1970, p. 7. 25. Allkemper parle de «Lebenserweiterung, befreite Lebenserfahrung (...) Wahrnehmung intensiver Lebensmöglichkeit », art. cité p. 134.

26. Norm, Abweichung und Krise. Über die Unvollendbarkeit des Realismus (1975), in : Das Verschwinden im Bild (1980), p. 119-120, non repris dans Werke.

27. Allkemper, art. cité, p. 142.

28. Das Schweigen der Sirenen, in : Franz Kafka, Sämtliche Erzählungen, hg. von Paul Raabe, Frankfurt a.M. (Fischer Bücherei), 1970, p. 305.

29. Transzendenz und scheinhafter Mehrwert. Zur Kategorie des Poetischen (1972), in: Werke, t.4, p. 302.

30. Ibid.

31. Allkemper, art. cité, p. 135.

32. Frédérique Craquelin, mémoire de maîtrise, Rouen 1994, p. 78.

\section{RÉSUMÉS}

L'article porte principalement sur deux romans de Dieter Wellershoff qui entretiennent des relations d'intertextualité complexes avec des œuvres de la «Weltliteratur »: Der Liebeswunsch (2000) avec Die Wahlverwandtschaften de Goethe, tandis que Die Sirene (1980) se nourrit d'essais théoriques de Blanchot et d'Adorno sur le récit homérique. Ces deux textes abordent des thèmes récurrents tant de l'œuvre romanesque que des essais théoriques de Wellershoff: la difficile quête individuelle du bonheur et la solitude. Der Liebeswunsch montre des héros " postmodernes » soumis à une contrainte sociale différente de celle pesant sur leurs ancêtres du xIX ${ }^{\mathrm{e}}$ siècle : non plus tant désormais l'étroitesse de la morale que la nécessité névrogène de réussir pleinement une vie individuelle, y compris sexuelle. Dans Die Sirene, le chant des sirènes est interprété comme une force intérieure à l'homme. Pour son héros, ébranlé par les appels téléphoniques d'une voix féminine anonyme, la perte de soi sera la condition de la régénération au moins 
partielle de soi. On retrouve une schéma dialectique de même type dans Der Liebeswunsch, où la recherche du bonheur engendre une contrainte dans laquelle le bonheur se perd. C'est ainsi une esthétique de l'écart significatif que Wellershoff met en œuvre avec l'utilisation des références intertextuelles.

Die zwei im Aufsatz analysierten Werke von Dieter Wellershoff sind der Weltliteratur mehrfach intertextuell verpflichtet: Der Liebeswunsch (2000) greift Elemente aus Goethes Wahlverwandtschaften auf, während der Novelle Die Sirene (1980) Thesen zugrundeliegen, die Blanchot und Adorno dem Homerschen Stoff zuschreiben. Beide Texte behandeln zudem Motive, die sowohl im literarischen, als auch im essayistischen Werk Wellershoffs relevant sind: die schwierige Glückssuche und das Alleinsein des Individuums in der heutigen Welt. Der Liebeswunsch läßt " postmoderne » Helden auftreten, die anderen gesellschaftlichen Zwängen unterliegen als ihre Vorfahren des 19. Jahrhunderts : nun weniger der Enge der herrschenden Moral als der sozial geforderten, neurosenerzeugenden Aufforderung zum vollen Lebens- bzw. Sexualgenuß. In Die Sirene wird Homers Gesang der Sirenen als Stimme aus dem eigenen Inneren des durch die Anrufe einer anonymen Frauenstimme zutiefst erschütterten Helden umgedeutet, für den sein Ich-Verlust die Vorausetzung zur partiellen Ich-Regenerierung bildet. Ein vergleichbarer dialektischer Ansatz zeigt sich auch in Der Liebeswunsch, wo die Glückssuche paradoxerweise das Glück zugrundezurichten droht. Mit dem Rückgriff auf intertextuelle Bezüge wird von Wellershoff somit eine Ästhetik der signifikanten Abweichung verwirklicht.

\section{AUTEUR}

\section{GÉRARD LAUDIN}

Université de Paris X-Nanterre 